\title{
Hydromagnetic waves in solar wind flow-structures
}

\begin{abstract}
P. S. Joarder
Physics Division, Variable Energy Cyclotron Centre (VECC), 1/AF Bidhan Nagar, Kolkata, 700064, India

Received 2 June 2000 / Accepted 28 November 2001

Abstract. Propagating magnetosonic waves in the observed fine structures of high-speed solar wind streams are theoretically examined, taking into account the non-parallel propagation of such waves. It is found that nonparallel propagation introduces new magnetoacoustic wavemodes, such as the forward-propagating slow surface modes and the backward-propagating fast surface modes that propagate outward in a direction away from the Sun; and the oppositely-propagating (slow and fast) body and surface modes that propagate towards the Sun in a frame co-moving with the ambient flow external to the solar wind flow-sheet. Such a wide variety of wavemodes is not available in a case where the propagation is purely parallel to the axis of the solar wind fine structure. It is argued here that the frequencies (in the satellite frame) of the outwardly propagating waves, that may transport photospheric/chromospheric oscillations into the heliospheric space, possibly lie in the range 6-100 mHz. Such a high-frequency band has not so far been explored for the detection of magnetosonic waves in the high-speed solar wind. Particularly interesting in the present study is the appearance of the sunward-propagating magnetosonic fast body waves. When viewed in a satellite (or solar) frame, such waves propagate towards the Sun provided that their angle of propagation exceeds a certain critical value, so that their propagation-vector is nearly normal to the solar equatorial plane. More high-resolution, multi-point in-situ observations are necessary to detect such sunward-propagating magnetoacoustic fast body waves in the fine flow structures of the solar wind at a distance of 1 AU from the Sun.
\end{abstract}

Key words. Sun: solar wind - magnetohydrodynamics - waves

\section{Introduction}

Recent observations made by two HELIOS spacecrafts have confirmed the existence of fine structures in highspeed solar wind flows. These structures are in the form of thin flow-sheets (or tubes) that are adjacent to each other with differences in their plasma parameters, such as their steady flow-speed along the magnetic field (Thieme et al. 1990). The structures may be separated by tangential discontinuities (TDs) in the magnetic field, across which the total (gas + magnetic) pressure is continuous. Such structures, therefore, are also called pressure balance structures (PBS; e.g. Tu \& Marsch 1995) in the literature. In fact, Parker (1963; see also McCraken \& Ness 1966; Mariani et al. 1973; Naugebauer 1981) first predicted the existence of such long and thin, coherent, spaghetti-like stream-structures as the constituent units of solar wind high-speed streams. These structures originate in the Sun, from where they spread out in the heliosphere, thus maintaining their identity even at a distance of $1 \mathrm{AU}$ from the Sun.

Recently, Nakariakov et al. (1996; which is a corrected version of Mann et al. 1992) have shown that the fine solar wind flow-structures may serve as waveguides for magnetoacoustic waves that may be excited by motion of the structures' foot points that are possibly anchored in the photospheric/chromospheric layer of the Sun. These authors modelled an individual flow-structure as a finitewidth slab (or sheet) with a reduced Alfven speed inside the slab and with an enhanced flow directed along the magnetic field parallel to the axis of the slab. This axis of the model flow-sheet was assumed to lie on the solar equatorial plane (see, for example, Burlaga 1995) pointing outwards from the position of the Sun. Nakariakov et al. (1996; referred hereafter as NRM96) then demonstrated that such a flow-sheet can sustain certain hydromagnetic wavemodes, namely, the backward, slow and fast body (kink and sausage) modes propagating along the sheetaxis in a direction away from the Sun. Such waves propagate backward relative to the flow inside the sheet with their phase-speed being smaller than the steady flow in the sheet (Nakariakov \& Roberts 1995). Identification of such backward-propagating waves is important as these waves are also the negative energy waves (Joarder et al. 1997b) that may introduce new instabilities, such as the dissipative, resonant or explosive instabilities (Cairns 1979; Craik \& Adam 1979; Craik 1985; Ryutova 1988; Hollweg et al. 1990; Ruderman \& Goossens 1995; Ruderman et al. 1996; Tirry et al. 1998; Ruderman \& Wright 1998; Andries et al. 2000 ) in solar wind fine structures. Such instabilities may

* e-mail: Joarder@veccal.ernet.in 
play dominant role in the amplification of waves and in the excitation of MHD turbulence in solar wind.

In their calculations, NRM96 did not consider the possibility of oblique propagation of waves; i.e. they ignored the component of propagation-vector in a direction normal to the solar equatorial plane containing both the axis and the direction of inhomogeneity of the model flowsheet. Such oblique propagation has recently been considered by Joarder \& Satya Narayanan (2000) in the context of the propagation of hydromagnetic surface waves in solar magnetic structures in the presence of steady shear flows. It was shown there that the inclusion of an oblique propagation-vector may introduce new surface waves that were not allowed by the shear-flow to propagate in a direction purely parallel to the field and the flow vectors. A similar property of non-parallel propagation of hydromagnetic waves was pointed out earlier by several authors (e.g. Uberoi 1982; Somasundaram \& Uberoi 1982; Rae \& Roberts 1983; Jain \& Roberts 1991; Joarder \& Roberts 1992) in different contexts and in the absence of shear flows. We also note that Tirry et al. (1998) and Andries et al. (2000) have recently analysed resonant flow instability of obliquely propagating hydromagnetic surface waves in a finite-width transition region between two uniform, zero plasma- $\beta$ magnetic media.

In view of continuing interest among the observers in the detection of hydromagnetic waves in solar wind (see the review by $\mathrm{Tu} \&$ Marsch 1995) as an important agent for heating and accelerating the solar wind plasma and for exciting solar wind MHD turbulence, we here extend the analysis of NRM96 by incorporating the important effect of oblique propagation of waves on the magnetoacoustic spectrum of a model solar wind flow-slab. The normal modes of a fine-stream structure that we here examine have a component of their wavevector in a direction normal to the solar equatorial plane. We find that, in addition to considerably modifying the backward waves reported by NRM96, oblique propagation also allows for new forward and backward propagating hydromagnetic body and surface modes that yield further complexity in the dynamics of solar wind fine structures. Specially, an oblique propagation-vector allows some of the magnetoacoustic waves of the solar wind flow-sheet to propagate in a direction opposite to that of the plasma flow, i.e. in a direction towards the Sun, when viewed in a frame co-moving with the ambient solar-wind flow external to the model flowsheet. Such oppositely propagating magnetoacoustic body and surface waves were entirely absent in the analysis of NRM96. It is here argued that some of the oppositely propagating body waves, with their propagation-vector nearly normal to the solar equatorial plane, may propagate towards the Sun even in a satellite frame (or, equivalently, in a frame fixed on the Sun; e.g. Kuijpers 1992; Cravens 1997). The existence of such sunward-propagating magnetoacoustic body waves is an important feature of the present calculation.

The plan of the paper is as follows. In Sect. 2, we describe an infinite slab (or sheet) model of the solar wind flow-structure and derive its magnetohydrodynamic dispersion relations. The properties of these dispersion relations are discussed in Sect. 3. The nature of waves and oscillations in a solar wind flow-sheet that may be relevant to observations, are presented in Sect. 4.

\section{The dispersion relations}

Following NRM96, we model the solar wind flow structure as a plane magnetic slab of width $2 a$. The slab is infinitely extended in the $y$ - and the $z$-directions with the slab boundaries being at $x= \pm a$. Both the magnetic fields (inside $\boldsymbol{B}_{\mathbf{0}}$ and outside $\boldsymbol{B}_{\mathbf{e}}$ ) and the steady flows (inside $\boldsymbol{U}_{\mathbf{0}}$ and outside $\boldsymbol{U}_{\mathbf{e}}$ ) are directed along the long-axis (the $z$-axis) of the slab. The sound speeds are $c_{\mathrm{s} 0}$ inside the slab and $c_{\mathrm{se}}$ outside; and the Alfven speeds are $v_{\mathrm{A} 0}$ and $v_{\mathrm{Ae}}$. The unperturbed gas densities $\left(\rho_{0}\right.$ inside the slab and $\rho_{\mathrm{e}}$ outside) are related by the condition of total pressure balance

$\frac{\rho_{\mathrm{e}}}{\rho_{0}}=\frac{2 c_{\mathrm{s} 0}^{2}+\gamma v_{\mathrm{A} 0}^{2}}{2 c_{\mathrm{se}}^{2}+\gamma v_{\mathrm{Ae}}^{2}} ;$

with the adiabatic index $\gamma=5 / 3$.

In this idealised model of a solar wind substructure, we may consider the plane containing the $x$ - and the $z$-axes to represent the solar equatorial plane with the negative $z$-direction being the direction towards the position of the Sun. The $y$ - axis is then directed along the normal to the solar equatorial plane and is parallel to the axis of rotation of the Sun. The effect of solar rotation, which is known to align the solar wind flow-tubes (or sheets) along an Archimedean spiral (Parker 1963) with the spiralling angle subtended with the $z$-direction at $1 \mathrm{AU}$ being around $88^{\circ}$ for the fast solar wind stream-tubes, has however been ignored in the present calculations. We expect that such a curvature of the flow-sheet may not have much bearing on the approximate propagation properties of the magnetohydrodynamic waves in a long and coherent fine structure of the fast solar wind stream. We note that similar idealisations have earlier been successfully employed to analyse the approximate properties of magnetoacoustic wavemodes in various solar magnetic structures, such as the solar coronal plasma loops (e.g. Edwin \& Roberts 1982, 1983; Roberts et al. 1984; Nakariakov et al. 1999) or the solar quiescent prominences (e.g. Joarder \& Roberts 1993; Joarder et al. 1997a).

We now linearise the ideal MHD equations about the above equilibrium with disturbances assumed to be of the form

$\Psi(x, y, z, t)=\Psi(x) \exp \left\{i \omega t+i k_{y} y+i k_{z} z\right\}$,

where, $\omega$ is the frequency of the disturbance and $k_{y}$, $k_{z}$ are the components of the wavevector $\boldsymbol{k}$ in the $y$ and the $z$-directions, respectively. We further define $\theta=$ $\tan ^{-1}\left|k_{y} / k_{z}\right|$, where $\theta$ is the acute angle $(0 \leq \theta<\pi / 2)$ subtended by the direction of the wavevector $\boldsymbol{k}$ with the $z-$ axis. Here, $\theta$ is measured in anticlockwise direction when 
both $k_{y}$ and $k_{z}$ are of same sign and in a clockwise direction when they are of opposite sign.

Elimination from the linearised equations of all variables except $v_{x}(x)$, the amplitude of the $x$-component of velocity, yields the second order ordinary differential equation (e.g. Joarder \& Roberts 1992; Joarder \& Satya Narayanan 2000)

$\frac{\mathrm{d}^{2} v_{x}}{\mathrm{~d} x^{2}}-k_{z}^{2} M_{(0, \mathrm{e})}^{2} v_{x}=0$,

where

$M_{(0, \mathrm{e})}^{2}=\frac{\left(v_{\mathrm{c}(0, \mathrm{e})}^{+^{2}}(\theta)-\Omega_{(0, \mathrm{e})}^{2}\right)\left(v_{\mathrm{c}(0, \mathrm{e})}^{-^{2}}(\theta)-\Omega_{(0, \mathrm{e})}^{2}\right)}{\left(v_{\mathrm{A}(0, \mathrm{e})}^{2}+c_{\mathrm{s}(0, \mathrm{e})}^{2}\right)\left(c_{\mathrm{T}(0, \mathrm{e})}^{2}-\Omega_{(0, \mathrm{e})}^{2}\right)}$

and $\Omega_{(0, \mathrm{e})} \equiv\left(\omega / k_{z}-U_{(0, \mathrm{e})}\right)$ are the Doppler-shifted, fieldaligned phase-speeds in the two media. The speeds

$c_{\mathrm{T}(0, \mathrm{e})}=c_{\mathrm{s}(0, \mathrm{e})} v_{\mathrm{A}(0, \mathrm{e})} /\left(c_{\mathrm{s}(0, \mathrm{e})}^{2}+v_{\mathrm{A}(0, \mathrm{e})}^{2}\right)^{1 / 2}$

are the magnetoacoustic "cusp-speeds" (e.g. Roberts 1981a) in the two media inside and outside the slab. The quantities $v_{\mathrm{c}(0, \mathrm{e})}^{ \pm}$are given by (Joarder \& Roberts 1992)

$$
\begin{aligned}
& v_{\mathrm{c}(0, \mathrm{e})}^{ \pm}(\theta)=\left\{\frac{1}{2}\left(v_{\mathrm{A}(0, \mathrm{e})}^{2}+c_{\mathrm{s}(0, \mathrm{e})}^{2}\right) \sec ^{2} \theta \pm \frac{1}{2}\right. \\
& \left.\times\left[\left(v_{\mathrm{A}(0, \mathrm{e})}^{2}+c_{\mathrm{s}(0, \mathrm{e})}^{2}\right)^{2} \sec ^{4} \theta-4 v_{\mathrm{A}(0, \mathrm{e})}^{2} c_{\mathrm{s}(0, \mathrm{e})}^{2} \sec ^{2} \theta\right]^{1 / 2}\right\}^{1 / 2},
\end{aligned}
$$

that are the magnetoacoustic speeds along the magnetic fields $\boldsymbol{B}_{(\mathbf{0}, \mathbf{e})}$, with "+" referring to the fast waves and "-" referring to the slow waves. For typical conditions in a solar wind flow-structure, we consider a condition obtained in HELIOS observations at 1 AU by Thieme et al. (1990). This condition is $v_{\mathrm{Ae}}>c_{\mathrm{se}}$ and $v_{\mathrm{A} 0}=c_{\mathrm{S} 0}$. In this situation, $v_{\mathrm{ce}}^{+}(\theta)$ approaches $v_{\mathrm{Ae}}, v_{\mathrm{ce}}^{-}(\theta)$ approaches $c_{\mathrm{se}}$ and $v_{\mathrm{c} 0}^{+}(\theta) \approx v_{\mathrm{c} 0}^{-}(\theta)$ approaches $v_{A 0}$ for a vanishingly small angle of propagation $\theta$. As $\theta$ approaches $\pi / 2, v_{\text {ce }}^{-}(\theta)$ approaches $c_{\mathrm{Te}}, v_{\mathrm{c} 0}^{-}(\theta)$ approaches $c_{\mathrm{T} 0}$, whereas $v_{\mathrm{ce}}^{+}(\theta)$ and $v_{\mathrm{c} 0}^{+}(\theta)$ become infinitely large. For intermediate values of the angle of propagation $\theta$, we have $v_{\mathrm{ce}}^{+}(\theta)>v_{\mathrm{Ae}}>c_{\mathrm{se}}>$ $v_{\mathrm{ce}}^{-}(\theta)>c_{\mathrm{Te}}$, and $v_{\mathrm{c} 0}^{+}(\theta)>v_{\mathrm{A} 0}=c_{\mathrm{s} 0}>v_{\mathrm{c} 0}^{-}(\theta)>c_{\mathrm{T} 0}$, respectively. We note that the condition considered here to be representative of the situation in a solar wind flowsheet is rather restrictive. In reality, different regions of the flow-structure at varying distances from the Sun may possess different plasma properties. Moreover, the Alfven speed and the acoustic speed of the plasma in a solar wind flow-sheet may not be exactly equal even at a distance of 1 AU from the Sun. Whatever be the case, the main purpose of the present study is to examine the effect of nonparallel propagation on the results obtained by NRM96 who considered the condition observed by Thieme et al. (1990) as the typical condition obtained in a solar wind flow-structure.

In the following, we restrict ourselves only to the normal modes of oscillations (Rae \& Roberts 1983) of the slab (or sheet) with the velocity amplitudes $v_{x}(x)$ supposed evanescent away from the slab (in $|x|>a$ ), implying $M_{\mathrm{e}}^{2}>0$. Considering further that both the normal component of displacement and the perturbation in total (gas + magnetic) pressure are continuous across the structure boundaries $x= \pm a$, we finally arrive at the dispersion relations

$$
\begin{aligned}
\left(\frac{\rho_{\mathrm{e}}}{\rho_{0}}\right)\left(v_{\mathrm{Ae}}^{2}-\Omega_{\mathrm{e}}^{2}\right) M_{0}+ & \left(v_{\mathrm{A} 0}^{2}-\Omega_{0}^{2}\right) M_{\mathrm{e}} \\
& \times\left(\begin{array}{c}
\tanh \\
\operatorname{coth}
\end{array}\right)\left(\left|k_{z} a\right| M_{0}\right)=0,
\end{aligned}
$$

where the choice of the "tanh" function represents symmetric (with respect to the $z$-axis) solutions and the choice of the "coth" function represents anti-symmetric solutions of Eq. (2). These are the kink and sausage modes, respectively (Roberts 1981b; Edwin \& Roberts 1982). In the absence of steady flows $\left(U_{\mathrm{e}}=U_{0}=0\right)$, Eq. (7) reduces to the dispersion relations obtained by Joarder \& Roberts (1992). For purely parallel propagation $\left(\theta=0^{\circ}\right)$ of the waves, Eq. (7) reduces to the dispersion relations derived by Nakariakov \& Roberts (1995). Finally, for $M_{0}^{2}>0$, corresponding to evanescent waves inside the slab, and in the short wavelength limit $\left|k_{z} a\right|>>1$, the present dispersion relations approach the dispersion relation obtained by Joarder \& Satya Narayanan (2000) for the surface waves at a single interface of discontinuity between two magnetic media in the presence of steady shear-flows.

We also note that Eqs. (6) and (7) can describe wavemodes propagating either in the direction of the solar wind flow (with $k_{z}>0$ ) or in a direction opposite to the flow (with $k_{z}<0$ ), as viewed in a frame co-moving with the external flow $\boldsymbol{U}_{\mathbf{e}}$. The angle of propagation $\theta$ may be given by the acute $(0 \leq \theta<\pi / 2)$ angle between the direction of the wavevector $\boldsymbol{k}$ and the $z$-axis in both the cases. In the following, we further assume $k_{y}>0$ simply for the sake of convenience, although the results that we obtain remain equally valid in the case $k_{y}<0$ for waves propagating in a direction opposite to the solar rotation vector along the normal to the solar equatorial plane.

\section{Properties of the dispersion relations}

To describe the nature of normal modes that are the solutions of the dispersion relations (7), we first choose a convenient frame of reference co-moving with the external flow, so that $U_{\mathrm{e}}=0, \Omega_{\mathrm{e}}=c \equiv \omega / k_{z}$. In this particular frame, the solutions of Eq. (7) that satisfy the condition of lateral evanescence $\left(M_{\mathrm{e}}^{2}>0\right)$ must have their field-aligned phase-speed $c$ such that either $|c|<c_{\mathrm{Te}}$, or, $v_{\text {ce }}^{-}(\theta)<|c|<v_{\text {ce }}^{+}(\theta)$. Following Roberts (1981a,b), we further classify these normal modes as the surface $\left(M_{0}^{2}>0\right)$ and body $\left(M_{0}^{2}<0\right)$ modes. Equation (4) then suggests that the modes with $c<\left(c_{\mathrm{T} 0}+U_{0}\right)$, or, $\left(v_{\mathrm{c} 0}^{-}(\theta)+U_{0}\right)<$ $c<\left(v_{\mathrm{c} 0}^{+}(\theta)+U_{0}\right)$, or, $\left(U_{0}-v_{\mathrm{c} 0}^{+}(\theta)\right)<c<\left(U_{0}-v_{\mathrm{c} 0}^{-}(\theta)\right)$ are surface modes. Similarly, modes with $\left(c_{\mathrm{T} 0}+U_{0}\right)<$ $c<\left(v_{\mathrm{c} 0}^{-}(\theta)+U_{0}\right)$, or, $\left(U_{0}-v_{\mathrm{c} 0}^{-}(\theta)\right)<c<\left(U_{0}-c_{\mathrm{T} 0}\right)$, or, $c<\left(U_{0}-v_{\mathrm{c} 0}^{+}\right)$are body modes. For surface mode solutions 
of Eq. (7), the field-aligned phase-speed $c$ satisfies an additional criterion that the factors $\left(v_{\mathrm{Ae}}^{2}-c^{2}\right)$ and $\left(v_{\mathrm{A} 0}^{2}-\Omega_{0}^{2}\right)$ must be of opposite signs (Nakariakov \& Roberts 1995).

It is convenient to define here six critical angles of propagation $\theta_{\mathrm{c} 1}$ to $\theta_{\mathrm{c} 6}$ that are given as the solutions of the equations

$$
\begin{aligned}
v_{\mathrm{c} 0}^{+}(\theta)= & U_{0},\left(\theta=\theta_{\mathrm{c} 1}\right), \\
v_{\mathrm{ce}}^{+}(\theta)= & \left(v_{\mathrm{c} 0}^{-}(\theta)+U_{0}\right),\left(\theta=\theta_{\mathrm{c} 2}\right), \\
v_{\mathrm{c} 0}^{+}(\theta)= & \left(c_{\mathrm{Te}}+U_{0}\right),\left(\theta=\theta_{\mathrm{c} 3}\right), \\
v_{\mathrm{c} 0}^{+}(\theta)= & \left(v_{\mathrm{ce}}^{-}(\theta)+U_{0}\right),\left(\theta=\theta_{\mathrm{c} 4}\right), \\
\cos \theta= & \left(\rho_{0} / \rho_{\mathrm{e}}\right) \\
& \times \frac{\left(U_{0}^{2}-v_{\mathrm{A} 0}^{2}\right)\left(U_{0}^{2}-c_{\mathrm{T} 0}^{2}\right)^{1 / 2}\left(v_{\mathrm{A} 0}^{2}+c_{\mathrm{s} 0}^{2}\right)^{1 / 2}}{v_{\mathrm{Ae}}^{2}\left(v_{\mathrm{c} 0}^{+2}(\theta)-U_{0}^{2}\right)^{1 / 2}\left(U_{0}^{2}-v_{\mathrm{c} 0}^{-2}(\theta)\right)^{1 / 2}}, \\
& \left(\theta=\theta_{\mathrm{c} 5}\right)
\end{aligned}
$$

and

$v_{\mathrm{c} 0}^{+}(\theta)=\left(v_{\mathrm{Ae}}+U_{0}\right),\left(\theta=\theta_{\mathrm{c} 6}\right)$.

The modal structure of the flow-sheet changes considerably as the angle of propagation $\theta$ exceeds any of these six critical angles, as we will see in the following.

To discuss the behaviour of solutions of the dispersion relations (7), we first note that the observed average values of various physical speeds in and out of the solar wind flow-structure at a distance of $1 \mathrm{AU}$ and in the aforesaid frame of reference (co-moving with the external flow at a speed of $655 \mathrm{~km} \mathrm{~s}^{-1}$ ) are given as $v_{\mathrm{A} 0}=c_{\mathrm{s} 0}=65 \mathrm{~km} \mathrm{~s}^{-1}$ $v_{\mathrm{Ae}}=100 \mathrm{~km} \mathrm{~s}^{-1}, c_{\mathrm{se}}=70 \mathrm{~km} \mathrm{~s}^{-1}$ and $U_{0}=95 \mathrm{~km} \mathrm{~s}^{-1}$ (Thieme et al. 1990). These values have also been considered by NRM96 (see also, Mann et al. 1992) in their calculations for purely parallel propagation of magnetoacoustic waves in a solar wind flow-structure. We simply consider these observed values to examine the effect of oblique propagation on the wavemodes. In such a case, Eqs. (1) and (8) give $\left(\rho_{\mathrm{e}} / \rho_{0}\right)=0.583, \theta_{\mathrm{c} 1}=32.13^{\circ}$, $\theta_{\mathrm{c} 2}=39.66^{\circ}, \theta_{\mathrm{c} 3}=54.88^{\circ}, \theta_{\mathrm{c} 4}=55.43^{\circ}, \theta_{\mathrm{c} 5}=61.27^{\circ}$ and $\theta_{\mathrm{c} 6}=62.73^{\circ}$, respectively. The observed values for the physical speeds satisfy the condition $\left(v_{\mathrm{A} 0}+U_{0}\right)=$ $\left(c_{\mathrm{s} 0}+U_{0}\right)>\left(c_{\mathrm{T} 0}+U_{0}\right)>v_{\mathrm{Ae}}>c_{\mathrm{se}}>c_{\mathrm{Te}}>\left(U_{0}-c_{\mathrm{T} 0}\right)>$ $\left(U_{0}-v_{\mathrm{A} 0}\right)$. For this ordering of parameters, normal mode solutions of Eq. (7) exist only in certain intervals of the longitudinal phase velocity $c$, depending on whether the direction of the longitudinal propagation-vector is parallel (implying $k_{z}>0$ ) or antiparallel (implying $k_{z}<0$ ) to the flow-vector $\boldsymbol{U}_{0}$. These two cases of longitudinal propagation are examined in detail in the following.

\subsection{Waves propagating in the direction of the flow $\left(k_{z}>0\right)$ :}

Waves belonging to this category propagate in a direction away from the Sun. Normal mode solutions of Eq. (7), that consist of such outwardly propagating waves, exist only in three propagation windows, namely, (i) $v_{\mathrm{Ae}}<$ $c<v_{\mathrm{ce}}^{+}(\theta)$, (ii) $\left(U_{0}-v_{\mathrm{c} 0}^{-}(\theta)\right)<c<\left(U_{0}-c_{\mathrm{T} 0}\right)$ and

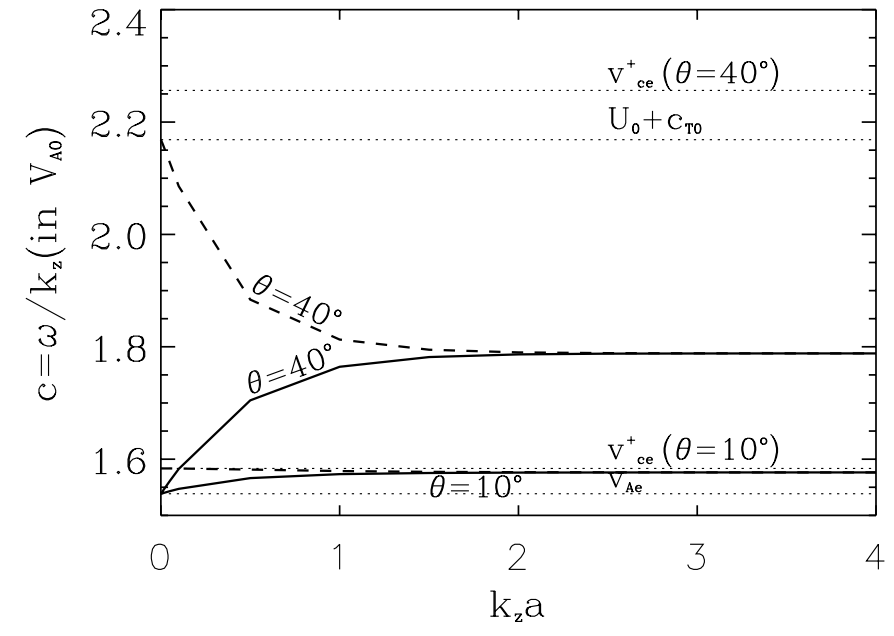

Fig. 1. Variation of the field-aligned phase-speed $c$ (in units of $v_{\mathrm{A} 0}$ ) with dimensionless wavenumber $k_{z} a$ for the (externally fast) magnetoacoustic slow surface modes in the flow-sheet in a case when $v_{\mathrm{A} 0}=c_{\mathrm{s} 0}=65 \mathrm{~km} \mathrm{~s}^{-1}, v_{\mathrm{Ae}}=1.539 v_{\mathrm{A} 0}, c_{\mathrm{se}}=$ $1.077 v_{\mathrm{A} 0}, U_{\mathrm{e}}=0, U_{0}=1.462 v_{\mathrm{A} 0}$ and for two arbitrarilychosen angles of propagation $\theta=10^{\circ}\left(<\theta_{\mathrm{c} 2}\right)$ and $\theta=40^{\circ}(>$ $\left.\theta_{\mathrm{c} 2}\right)$. The continuous curves denote the kink surface modes and the broken curves denote the sausage surface modes.

(iii) $0<c<\left(U_{0}-v_{\mathrm{A} 0}\right)$. We now proceed to briefly discuss the nature of wavemodes in these three intervals of the longitudinal phase-speed $c$.

(i) $v_{\mathrm{Ae}}<c<v_{\mathrm{ce}}^{+}(\theta)$ : when $\theta<\theta_{\mathrm{c} 2}$, so that $v_{\mathrm{ce}}^{+}(\theta)<$ $\left(v_{\mathrm{c} 0}^{-}(\theta)+U_{0}\right)$ (see Eq. (8)), only one band of surface modes consisting of the (externally fast) magnetoacoustic slow (kink and sausage) surface modes exists in this region that owes its existence solely to a non-zero angle of propagation $\theta$. These surface modes are shown in Fig. 1 for two different values of $\theta$, namely, $\theta=10^{\circ}(<$ $\left.\theta_{c 2}\right)$ and $\theta=40^{\circ}\left(>\theta_{\mathrm{c} 2}\right)$, respectively. This figure shows that, for $\theta>\theta_{\mathrm{c} 2}$, the field-aligned phase-speed $c$ of the (externally fast) slow surface modes satisfy a condition $v_{\mathrm{Ae}}<c<\left(c_{\mathrm{T} 0}+U_{0}\right)$. For such an angle of propagation $\theta>\theta_{c 2}$, two new bands of modes, namely, the (externally fast) magnetoacoustic slow body (kink and sausage) modes and the (externally fast) magnetoacoustic fast (kink and sausage) surface modes appear with their phase-speed $c$ lying in the intervals $\left(c_{\mathrm{T} 0}+U_{0}\right)<c<\left(v_{\mathrm{c} 0}^{-}(\theta)+U_{0}\right)$ and $\left(v_{\mathrm{c} 0}^{-}(\theta)+U_{0}\right)<c<v_{\mathrm{ce}}^{+}(\theta)$, respectively. Figure 2 shows the fundamental, (externally fast) slow body (kink and sausage) modes and the (externally fast) fast sausage surface mode, all for a particular angle of propagation $\theta=40^{\circ}\left(>\theta_{\mathrm{c} 2}\right)$. We may add here that the fundamental, slow body kink mode transforms into an (externally fast) fast kink surface mode for wavenumber $k_{z} a \geq 31.00$, for which the field-aligned phase-speed $c$ of the mode satisfies a condition $c \geq\left(v_{\mathrm{c} 0}^{-}\left(\theta>\theta_{\mathrm{c} 2}\right)+U_{0}\right)$. Such large values of the dimensionless wavenumber $k_{z} a$ is out of the range shown in Fig. 2.

As $\theta$ approaches $\pi / 2$, the propagation band for the slow body waves collapses to a single line $c=c_{\mathrm{T} 0}+U_{0}$; whereas the propagation band for the fast surface waves 


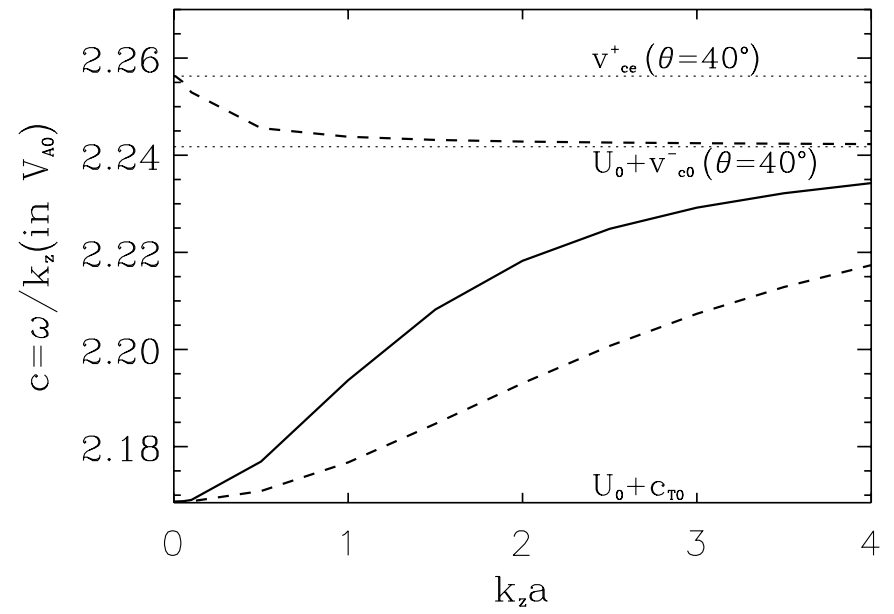

Fig. 2. Field-aligned phase-speed $c$ vs. dimensionless wavenumber $k_{z} a$ for the fundamental, (externally fast) magnetoacoustic slow body kink mode (continuous curve), the fundamental, (externally fast) magnetoacoustic slow body sausage mode (lower broken curve) and the (externally fast) magnetoacoustic fast sausage surface mode (upper broken curve) in the flow-sheet for an angle of propagation $\theta=40^{\circ}\left(>\theta_{\mathrm{c} 2}\right)$. Numerical values of various physical speeds chosen to draw this diagram are the same as in Fig. 1. The fundamental slow body kink mode transforms into a fast kink surface mode at a large value of the wavenumber $k_{z} a$ that is out of the range considered in this figure.

changes to $\left(c_{\mathrm{T} 0}+U_{0}\right)<c<\left(v_{\mathrm{A} 0}+U_{0}\right)$ in this limit of near-normal propagation with respect to the axis of the slab (sheet).

(ii) $\left(U_{0}-v_{\mathrm{c} 0}^{-}(\theta)\right)<c<\left(U_{0}-c_{\mathrm{T} 0}\right)$ : modes appearing in this propagation band are the backward-propagating, magnetoacoustic slow body (kink and sausage) modes that were found by NRM96 in a propagation-window $\left(U_{0}-\right.$ $\left.v_{\mathrm{A} 0}\right)<c<\left(U_{0}-c_{\mathrm{T} 0}\right)$ in the case of a purely parallel $\left(\theta=0^{\circ}\right)$ propagation along the direction of the flow. Such backward body modes are shown in Fig. 3 for two different angles of propagation $\theta$. The propagation-band for these slow body modes narrows down with the increase of the angle $\theta$ to ultimately collapse to a single line $c=\left(U_{0}-c_{\mathrm{T} 0}\right)$ as $\theta$ approaches $\pi / 2$.

(iii) $0<c<\left(U_{0}-v_{\mathrm{A} 0}\right)$ : magnetoacoustic modes that are available in this interval of phase-speed $c$ are all displayed in Fig. 4 for different values of the angle of propagation $\theta$ with respect to the direction of the steady flow $\boldsymbol{U}_{0}$. This figure shows that, for $\theta=10^{\circ}\left(<\theta_{\mathrm{c} 1}\right.$; see Eq. (8)), we obtain the backward-propagating, magnetoacoustic fast body (kink and sausage) modes and the backward-propagating, magnetoacoustic fast (kink and sausage) surface modes with their phase-speeds lying in the propagation-regions $0<c<\left(U_{0}-v_{\mathrm{c} 0}^{+}(\theta)\right)$ and $\left(U_{0}-v_{\mathrm{c} 0}^{+}(\theta)\right)<c<\left(U_{0}-\right.$ $\left.v_{\mathrm{A} 0}\right)$, respectively. We note here that only the backwardpropagating, fast body modes were obtained by NRM96 in the propagation region $0<c<\left(U_{0}-v_{\mathrm{A} 0}\right)$ in the case of a purely parallel propagation with $\theta=0^{\circ}$. These body modes have critical wavenumbers $k_{\mathrm{z} 0}\left(\theta<\theta_{\mathrm{c} 1}\right)$ at which

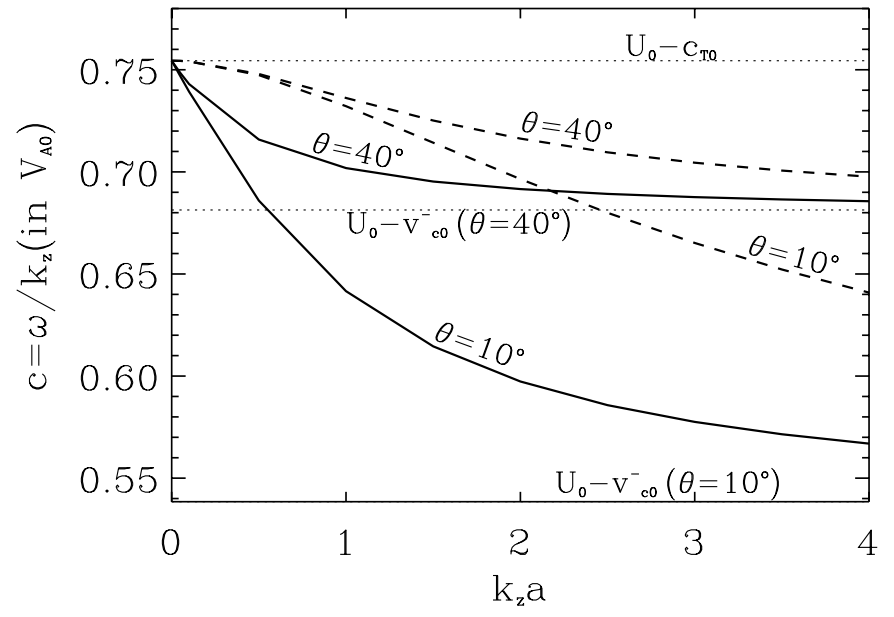

Fig. 3. Field-aligned phase-speed $c$ vs. dimensionless wavenumber $k_{z} a$ for the fundamental, backward-propagating, magnetoacoustic slow body kink mode (denoted by the continuous curves) and the fundamental, backward-propagating, magnetoacoustic slow body sausage mode (denoted by the broken curves) for two different values of the angle of propagation $\theta$, namely, $\theta=10^{\circ}$ and $\theta=40^{\circ}$. Numerical values of various physical speeds are chosen to be the same as in Fig. 1.

their phase-speed $c$ drops to zero. Such critical wavenumbers are given by:

$k_{\mathrm{z} 0}(\theta)=\frac{1}{a N_{0}(c=0)}\left(\begin{array}{c}\tan ^{-1}(\Lambda) \\ \cot ^{-1}(-\Lambda)\end{array}\right) ;$

where,

$\Lambda=\frac{\rho_{\mathrm{e}} N_{0}(c=0) v_{\mathrm{Ae}}^{2} \cos \theta}{\rho_{0}\left(U_{0}^{2}-v_{\mathrm{A} 0}^{2}\right)}$

and

$N_{0}^{2}(c=0)=\frac{U_{0}^{4}}{v_{\mathrm{A} 0}^{2}\left(2 U_{0}^{2}-v_{\mathrm{A} 0}^{2}\right)}-\sec ^{2} \theta$.

In Eq. (9), the " $\tan ^{-1}$ " function corresponds to the kink mode and the " $\cot ^{-1}$ " function corresponds to the sausage mode. The fast body modes with their wavenumbers smaller than these critical wavenumbers are no longer the backward- propagating modes. Such a mode propagates in a direction opposite to the flow (i.e. with $k_{z}<0$ ) with its wavevector subtending an acute angle $\theta$ (measured in a clockwise direction) with the vector $-\boldsymbol{U}_{\mathbf{0}}$ as we will see in Sect. 3.2.

The phase-speed $c$ of the backward-propagating, fast body sausage modes approaches $\left(U_{0}-v_{\mathrm{c} 0}^{+}(\theta)\right)$ as $k_{z} a$ approaches infinity; whereas, the fundamental, backwardpropagating, fast body kink mode transforms into the backward-propagating, fast kink surface mode at an intermediate value of the wavenumber $k_{z} a$, at which the longitudinal phase-speed $c$ for the kink mode equals $\left(U_{0}-\right.$ $\left.v_{\mathrm{c} 0}^{+}(\theta)\right)$.

As $\theta$ increases through its critical value $\theta_{\mathrm{c} 1}$, so that $\left(U_{0}-v_{\mathrm{c} 0}^{+}(\theta)\right)<0$, the backward-propagating fast body modes disappear leaving only the backward-propagating, fast (kink and sausage) surface modes in the propagation 


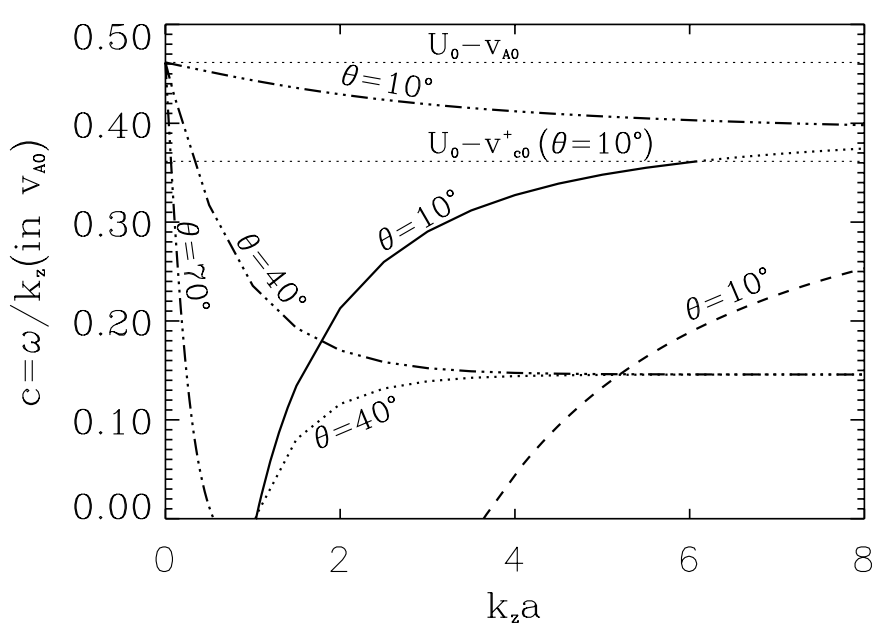

Fig. 4. Field-aligned phase-speed $c$ vs. dimensionless wavenumber $k_{z} a$ for magnetoacoustic modes appearing in the propagation window $0<c<\left(U_{0}-v_{\mathrm{A} 0}\right)$ for different angles of propagation $\theta$, namely, $\theta=10^{\circ}, 40^{\circ}$ and $70^{\circ}$ that are representatives of the situations $\theta<\theta_{\mathrm{c} 1}, \theta_{\mathrm{c} 1}<\theta<\theta_{\mathrm{c} 5}$, and $\theta>\theta_{\mathrm{c} 5}$, respectively. Here, a continuous (-) curve denotes the fundamental, backward-propagating, magnetoacoustic fast body kink mode, a broken $\left(--_{-}-\right)$curve denotes the fundamental, backward-propagating, magnetoacoustic fast body sausage mode, a dotted (. . . . . .) curve denotes a backward-propagating, magnetoacoustic fast kink surface mode and a dotted-dashed (- ......-) curve denotes a backward-propagating, magnetoacoustic fast sausage surface mode. Note that, for $\theta<\theta_{\mathrm{c} 1}$, the backward fast body kink mode transforms into a backward fast kink surface mode at an intermediate value of the wavenumber at which the phase-speed $c$ of the mode exceeds $\left(U_{0}-v_{\mathrm{c} 0}^{+}\left(\theta<\theta_{\mathrm{c} 1}\right)\right)$. The backward body modes disappear for propagation angles exceeding $\theta_{\mathrm{c} 1}$; while the backward fast kink surface mode disappears as $\theta$ exceeds the critical angle $\theta_{\mathrm{c} 5}$. Also, note the existence of critical wavenumbers at which the phase-speed $c$ of the corresponding wavemodes drops to zero; see discussion in the main text. Numerical values for various physical speeds chosen to draw this diagram are the same as in Fig. 1.

window $0<c<\left(U_{0}-v_{\mathrm{A} 0}\right)$. This situation is also shown in Fig. 4 for an arbitrarily-chosen angle of propagation $\theta\left(>\theta_{\mathrm{c} 1}\right)=40^{\circ}\left(<\theta_{\mathrm{c} 5} ;\right.$ see Eq. (8)). Note that the fast kink surface mode is a backward-propagating mode only for wavenumbers greater than a certain critical wavenumber $k_{\mathrm{z} 0 \text {, kink }}\left(\theta_{\mathrm{c} 1}<\theta<\theta_{\mathrm{c} 5}\right)$ at which the phase velocity of this kink surface mode drops to zero. This critical wavenumber is given by the relation for the kink mode in Eq. (9) after replacing $N_{0}$ by $M_{0}=i N_{0}$ and $\tan ^{-1}(\Lambda)$ by $-i \tanh ^{-1}(i \Lambda)$, respectively.

As $\theta$ exceeds the critical angle $\theta_{\mathrm{c} 5}$, the backwardpropagating kink surface mode completely disapears. Only the backward mode, that survives such an angle of propagation $\theta>\theta_{\mathrm{c} 5}$ in the propagation window $0<c<$ $\left(U_{0}-V_{\mathrm{A} 0}\right)$, is the fast sausage surface mode that now possesses a critical wavenumber $k_{\mathrm{z} 0 \text {, sausage }}\left(\theta>\theta_{\mathrm{c} 5}\right)$; see the curve in Fig. 4 that corresponds to $\theta=70^{\circ}$. For a wavenumber greater than this critical wavenumber, the backward fast sausage surface mode transforms itself into an oppositely-propagating, magnetoacoustic fast sausage surface mode discussed in Sect. 3.2. This critical wavenumber for the backward fast sausage surface mode may again be obtained from the relation for the sausage mode in Eq. (9) after replacing $N_{0}$ by $i M_{0}$ and $\cot ^{-1}(-\Lambda)$ by $i \operatorname{coth}^{-1}(-i \Lambda)$, respectively.

\subsection{Waves propagating opposite to the direction of the flow $\left(k_{z}<0\right)$ :}

In their calculations with a purely longitudinal wavevector (i.e. with $k_{y}=0$ ), NRM96 did not obtain magnetoacoustic waves propagating in the negative $z$-direction in a frame co-moving with the external flow $\boldsymbol{U}_{\mathbf{e}}$. This result seems to be an artifact of their choice of physical speeds inside a solar wind substructure, so that $v_{\mathrm{A} 0}=c_{\mathrm{s} 0}$. Although we here choose the same set of values for the physical parameters as the one considered by NRM96, the inclusion of a non-parallel propagation vector that yields $v_{\mathrm{c} 0}^{+}(\theta) \neq v_{\mathrm{ce}}^{-}(\theta)$ in our calculations allows for the existence of oppositely-propagating magnetosonic wavemodes that propagate in a direction opposite to the direction of the flow in a solar wind substructure with respect to a frame in which $U_{\mathrm{e}}=0$. The various propagation windows for such oppositely-propagating magnetoacoustic modes are discussed in the following.

(i) $-c_{\mathrm{Te}}<c<0$ : when $\theta<\theta_{\mathrm{c} 1}$, so that $\left(U_{0}-v_{\mathrm{c} 0}^{+}(\theta)\right)>0$, the wavemodes that appear in this propagation window are the oppositely-propagating, (externally slow) magnetoacoustic fast body (kink and sausage) modes that are shown in Fig. 5 for a particular angle of propagation $\theta=10^{\circ}\left(<\theta_{\mathrm{c} 1}\right)$ with respect to the vector $-\boldsymbol{U}_{\mathbf{0}}$. For such an angle of propagation $\theta<\theta_{c 1}$, both the fast body modes possess critical wavenumbers $\left|k_{\mathrm{z} 0}(\theta)\right|$ that are numerically the same as the critical wavenumbers pertaining to the backward-propagating, magnetoacoustic fast body (kink and sausage) modes displayed in Fig. 4 in Sect. 3.1. Figures 4 and 5 together demonstrate that, for a magnitude of the wavenumbers larger than critical wavenumbers, the oppositely-propagating, (externally slow) fast body modes are dragged along with the internal flow $\boldsymbol{U}_{\mathbf{0}}$ so that the kink and sausage body modes change their direction of propagation with respect to the direction of the flow to become backward-propagating, fast body kink and fast body sausage modes, respectively.

Such a critical wavenumber for the fast body sausage mode is removed for $\theta_{\mathrm{c} 1}<\theta<\theta_{\mathrm{c} 3}$ (see Eq. (8)), so that the longitudinal phase velocity $c$ of the mode asymptotically approaches $\left(U_{0}-v_{\mathrm{c} 0}^{+}(\theta)\right)$ for arbitrarily large magnitude of the dimensionless wavenumber $\left|k_{z} a\right|$. For such an angle of propagation $\theta_{\mathrm{c} 1}<\theta<\theta_{\mathrm{c} 3}$, the fundamental, fast body kink mode transforms into an oppositelypropagating, (externally slow) fast kink surface mode at an intermediate value of $\left|k_{z} a\right|$, for which the phase velocity of the kink mode equals $\left(U_{0}-v_{\mathrm{c} 0}^{+}(\theta)\right)$. Such behaviour of the oppositely-propagating, fast magnetosonic body and surface modes are displayed in Fig. 5 for an arbitrarily-chosen 


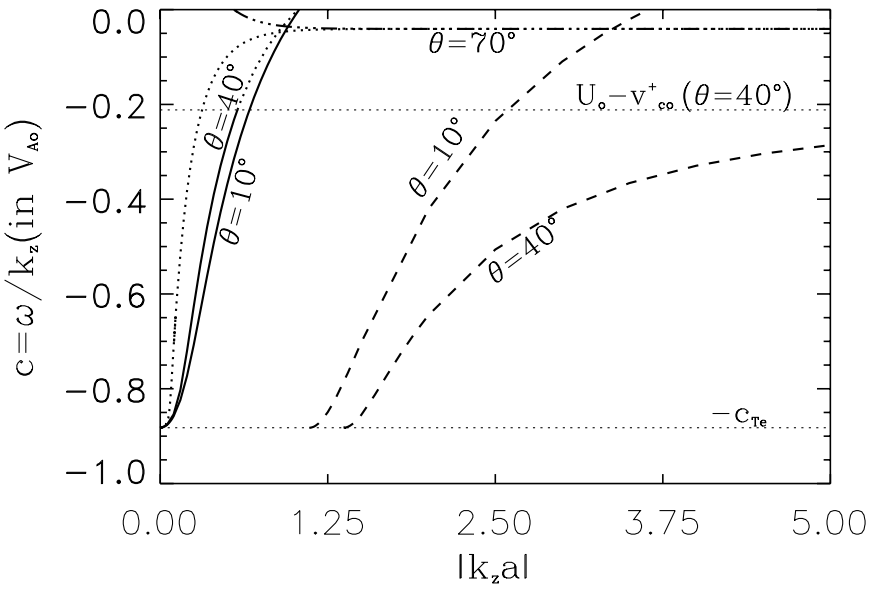

Fig. 5. Field-aligned phase-speed $c$ vs. the magnitude of the dimensionless wavenumber $\left|k_{z} a\right|$ for various oppositelypropagating, magnetosonic wavemodes that appear in the propagation-window $-c_{\mathrm{Te}}<c<0$ for different values of the angle of propagation $\theta$, namely, $\theta=10^{\circ}$ (representing the situation $0<\theta<\theta_{\mathrm{c} 1}$ ), $40^{\circ}$ (representing $\theta_{\mathrm{c} 1}<\theta<\theta_{\mathrm{c} 3}$ ) and $70^{\circ}$ (representing $\theta>\theta_{\mathrm{c} 5}$ ), respectively with respect to the flowvector $-\boldsymbol{U}_{\mathbf{0}}$. Here, a continuous (-) curve denotes the fundamental, (externally slow) fast body kink mode, a broken $\left(---_{-}\right)$curve denotes the fundamental, (externally slow) fast body sausage mode, a dotted $(. . .$.$) curve denotes the$ (externally slow) fast kink surface mode and a dotted-dashed $(-\cdots-\cdots-)$ curve denotes the (externally slow) fast sausage surface mode, respectively. Numerical values of various physical speeds are chosen to be the same as in Fig. 1. Note that, for $\theta_{\mathrm{c} 1}<\theta<\theta_{\mathrm{c} 3}$, the oppositely-propagating, fundamental, fast body kink mode transforms into an oppositely-propagating, fast kink surface mode at an intermediate value of $\left|k_{z} a\right|$, for which the phase-speed $c$ of the mode equals $\left(U_{0}-v_{\mathrm{c} 0}^{+}(\theta)\right)$.

angle of propagation $\theta\left(>\theta_{\mathrm{c} 1}\right)=40^{\circ}\left(<\theta_{\mathrm{c} 3}\right)$. This figure further shows that, for $0<\theta<\theta_{\mathrm{c} 3}$, the fundamental, fast body sausage mode possesses lower cut-off with the cut-off wavenumber given by

$\left|k_{\mathrm{zc}}\right|_{\text {sausage }}\left(\theta<\theta_{\mathrm{c} 3}\right)=\frac{\pi}{2 a N_{0}\left(c=-c_{\mathrm{Te}}\right)} ;$

where,

$N_{0}\left(c=-c_{\mathrm{Te}}\right)=i M_{0}\left(c=-c_{\mathrm{Te}}\right)$

with $M_{0}$ given by Eq. (4) of Sect. 2. For wavenumbers less than such cut-off wavenumber, the fundamental, fast body sausage mode changes into a leaky mode that drives magnetosonic waves into the medium external to the flowsheet and, therefore, decays in time; see the discussions on leaky modes in Roberts \& Webb (1979).

As the angle of propagation $\theta$ increases further, so that $\theta_{\mathrm{c} 3}<\theta<\theta_{\mathrm{c} 5}$ (see, Eq. (8)), the fast body modes completely disappear leaving only an oppositely-propagating, (externally slow) magnetoacoustic fast kink surface mode with its longitudinal phase-speed $c$ lying in the range $-c_{\mathrm{Te}}<c<0$. As $\theta$ exceeds its critical value $\theta_{\mathrm{c} 5}$, both the oppositely-propagating, (externally slow) magnetoacoustic fast kink and fast sausage surface modes appear in this propagation window with the sausage mode

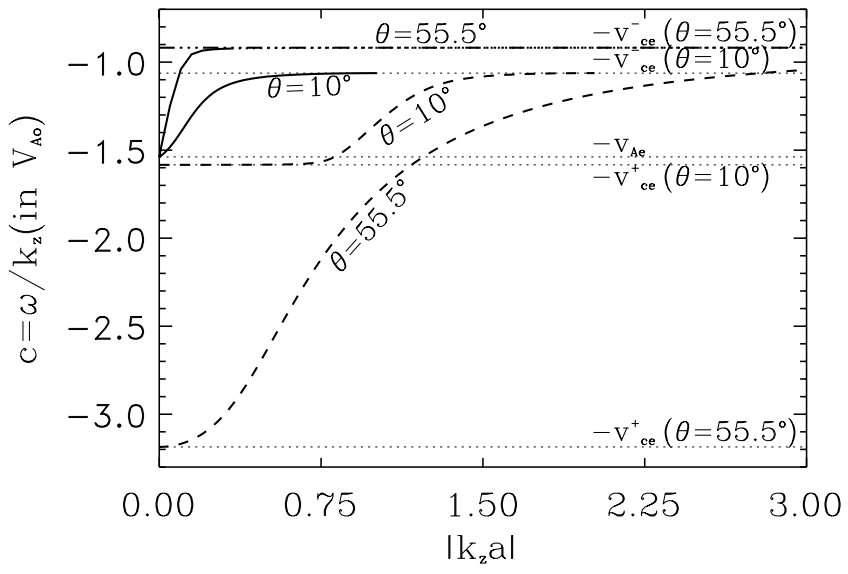

Fig. 6. Field-aligned phase-speed $c$ vs. the magnitude of the dimensionless wavenumber $\left|k_{z} a\right|$ for various oppositelypropagating modes, that appear in the interval of phase-speed $-v_{\mathrm{ce}}^{+}(\theta)<c<-v_{\mathrm{ce}}^{-}(\theta)$, for two different values of the angle of propagation $\theta$, namely, $\theta=10^{\circ}$ and $\theta=55.5^{\circ}$ that are the representatives of the situations $0<\theta<\theta_{\mathrm{c} 4}$ and $\theta_{\mathrm{c} 4}<\theta<\theta_{\mathrm{c} 6}$, respectively. Here, a continuous (fundamental, (externally fast) magnetoacoustic fast body kink mode, a broken (- - - - -) curve denotes the fundamental, (externally fast) magnetoacoustic fast body sausage mode, a dotted $(. . .$.$) curve denotes the (externally fast) magne-$ toacoustic fast kink surface mode, and a broken-dashed (- . . ...-) curve denotes the (externally fast) magnetoacoustic fast sausage surface mode, respectively. Note that, the curves denoting the oppositely-propagating, fast kink and fast sausage surface modes for $\theta=55.5^{\circ}$ are almost indistinguishable, both being almost coincident with the line $c=-v_{\mathrm{ce}}^{-}\left(\theta=55.5^{\circ}\right)$ in this figure. This is an artifact of the scale chosen to draw this diagram. Note also, that the oppositely-propagating, fundamental, fast body kink mode transforms into an oppositelypropagating, fast kink surface mode as the phase-speed $c$ of the kink mode exceeds a value $\left(U_{0}-v_{\mathrm{c} 0}^{+}\left(\theta=55.5^{\circ}\right)\right)$. This transition is not clearly shown in this figure. Numerical values of various physical speeds chosen to draw this diagram are the same as in Fig. 1.

having a critical wavenumber $\left|k_{\mathrm{z} 0 \text {, sausage }}\left(\theta>\theta_{\mathrm{c} 5}\right)\right|$. For wavenumbers smaller than this critical wavenumber, the sausage mode changes into a backward-propagating, magnetoacoustic fast sausage surface mode that was shown in Fig. 4. Figure 5 shows such oppositely-propagating, (externally slow) fast (kink and sausage) surface waves for an arbitrarily-chosen angle of propagation $\theta=70^{\circ}\left(>\theta_{\mathrm{c} 5}\right)$.

(ii) $-v_{\mathrm{ce}}^{+}(\theta)<c<-v_{\mathrm{ce}}^{-}(\theta)$ : when $0<\theta<\theta_{\mathrm{c} 4}$, so that $v_{\mathrm{c} 0}^{+}(\theta)<\left(U_{0}+v_{\mathrm{ce}}^{-}(\theta)\right)$ (cf. Eq. $\left.(8)\right)$, the wavemodes that are available in this propagation window are the oppositelypropagating, (externally fast) magnetoacoustic fast body (kink and sausage) modes. This particular case is shown in Fig. 6 for an arbitrarily-chosen angle of propagation $\theta=10^{\circ}$. This figure shows that, for $0<\theta<\theta_{\mathrm{c} 4}$, both the kink and sausage fast body modes have upper cut-offs with the cut-off wavenumbers given by

$$
\left|k_{\mathrm{zc}}\right|_{\text {kink }}\left(0<\theta<\theta_{\mathrm{c} 4}\right)=\frac{\pi}{2 a N_{0}\left(c=-v_{\mathrm{ce}}^{-}\left(0<\theta<\theta_{\mathrm{c} 4}\right)\right)},
$$




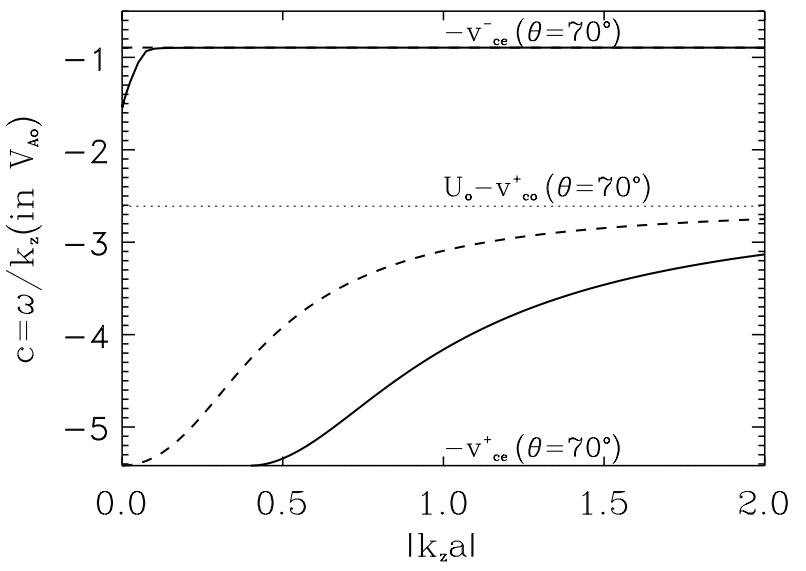

Fig. 7. Field-aligned phase-speed $c$ vs. magnitude of the dimensionless wavenumber $\left|k_{z} a\right|$ for the oppositely-propagating modes appearing in the propagation interval $-v_{\mathrm{ce}}^{+}(\theta)<c<$ $-v_{\text {ce }}^{-}(\theta)$, for a particular angle of propagation $\theta=70^{\circ}$ representing the case $\theta>\theta_{\mathrm{c} 6}$. In this figure, the upper continuous curve represents the (externally fast) fast kink surface mode and the lower continuous curve denotes the fundamental, (externally fast) fast body kink mode. Similarly, the upper broken curve denotes the (externally fast) fast sausage surface mode and the lower broken curve denotes the fundamental, (externally fast) fast body sausage mode. Note that, the curves representing the kink and sausage fast surface waves are almost overlapping, with their phase-speed being close to the value $-v_{\mathrm{ce}}^{-}(\theta)$, for most of the diagram except for small values of $\left|k_{z} a\right|$, where the two curves separate out from each other. Numerical values of various physical speeds chosen to draw this diagram are the same as in Fig. 1.

for the fundamental kink mode and

$\left|k_{\mathrm{zc}}\right|_{\text {sausage }}\left(0<\theta<\theta_{\mathrm{c} 4}\right)=\frac{\pi}{a N_{0}\left(c=-v_{\mathrm{ce}}^{-}\left(0<\theta<\theta_{\mathrm{c} 4}\right)\right)}$,

for the fundamental sausage mode, respectively. Here $N_{0}=i M_{0}$ (see Eq. (4) in Sect. 2).

As $\theta$ exceeds its critical value $\theta_{\mathrm{c} 4}$, so that $\theta_{\mathrm{c} 4}<\theta<\theta_{\mathrm{c} 6}$ implying $-v_{\mathrm{Ae}}<\left(U_{0}-v_{\mathrm{c} 0}^{+}(\theta)\right)<-v_{\mathrm{ce}}^{-}(\theta)$, the upper cut-offs for the (externally fast) fast body modes are removed. The phase-speed $c$ of the fundamental, fast body sausage mode now approaches $\left(U_{0}-v_{\mathrm{c} 0}^{+}\left(\theta_{\mathrm{c} 4}<\theta<\theta_{\mathrm{c} 6}\right)\right)$ for arbitrarily large magnitude of the wavenumber $\left|k_{z} a\right|$; whereas the fundamental, fast body kink mode transforms itself into an oppositely-propagating (externally fast) magnetoacoustic fast kink surface mode as its phasespeed $c$ exceeds $\left(U_{0}-v_{\mathrm{c} 0}^{+}(\theta)\right)$. In addition, we obtain an oppositely-propagating, (externally fast) magnetoacoustic fast sausage surface mode with its longitudinal phasespeed lying in the interval $\left(U_{0}-v_{\mathrm{c} 0}^{+}(\theta)\right)<c<-v_{\mathrm{ce}}^{-}(\theta)$. Such body and surface modes are displayed in Fig. 6 for an angle of propagation $\theta\left(>\theta_{\mathrm{c} 4}\right)=55.5^{\circ}\left(<\theta_{\mathrm{c} 6}\right)$.

Finally, in Fig. 7, we display the oppositelypropagating, (externally fast) fast magnetoacoustic body and surface modes as the angle $\theta$ exceeds its critical value $\theta_{\mathrm{c} 6}$. We find that the propagation bands for the body and surface modes are now distinctly separated with the body modes propagating in the region $-v_{\mathrm{ce}}^{+}\left(\theta>\theta_{\mathrm{c} 6}\right)<$ $c<\left(U_{0}-v_{\mathrm{c} 0}^{+}\left(\theta>\theta_{\mathrm{c} 6}\right)\right)$ and the surface modes propagating in the region $\left(U_{0}-v_{\mathrm{c} 0}^{+}\left(\theta>\theta_{\mathrm{c} 6}\right)\right)<c<-v_{\mathrm{ce}}^{-}\left(\theta>\theta_{\mathrm{c} 6}\right)$. We also note that the fundamental, fast body kink mode now has a lower cut-off with the cut-off wavenumber given by:

$$
\left|k_{\mathrm{zc}}\right|_{\text {kink }}\left(\theta>\theta_{\mathrm{c} 6}\right)=\frac{\pi}{2 a N_{0}\left(c=-v_{\mathrm{ce}}^{+}\left(\theta>\theta_{\mathrm{c} 6}\right)\right)} .
$$

\section{Waves in solar wind flow-structures}

Having described in general terms properties of the dispersion relations (7), we now set ourselves for examining waves in an individual solar wind flow-structure embedded in an ambient solar wind flow that may be detected in the in-situ observations by space satellites at a distance of 1 AU from the Sun. We find, from discussions given in the previous section, that the magnetosonic wavemodes that may be sustained by such a flow-sheet may propagate along the axis of the sheet either in an outward (positive $z^{-}$) direction or in an inward (negative $z^{-}$) direction, opposite to the internal flow $\boldsymbol{U}_{\mathbf{0}}$, in a frame co-moving with the flow external to the flow-sheet. We discuss these two cases of propagation separately in the following.

\subsection{Outwardly propagating waves}

Important periodicities of waves that may propagate in an outward direction in a solar wind flow-structure at $1 \mathrm{AU}$ are expected to be in the range $3-20 \mathrm{~min}$. This is because the solar wind flow-structures are believed to be the signatures of underlying magnetic structures, such as plumes and macrospicules in solar coronal holes (e.g. Parker 1963; Thieme et al. 1990; Velli et al. 1994; Del Zenna \& Velli 1999), the footpoints of which are firmly anchored at the solar photosphere/chromosphere in the form of thin magnetic fluxtubes (Spruit 1981; Spruit \& Roberts 1983). Several authors (cf. Hollweg 1982; Mann et al. 1992; Nakariakov et al. 1996) have, therefore, assumed that the ultimate source of outwardly propagating wave-energy in these fine flow-structures of solar wind may be the oscillatory motions in the photospheric/chromospheric layer of the Sun. We however note that, only a tiny fraction of such wave energy generated at these lowermost layers of solar atmosphere may ultimately reach the inner heliosphere, after appreciable loss suffered by downward reflection at the solar transition region and by various dissipative and nondissipative damping mechanisms in solar coronal holes. Such oscillatory motions exhibit periodicities in the range 3-15 min in solar photosphere (e.g. Christensen-Dalsgaard 1992); whereas, in solar chromosphere, velocity oscillations possess periods of around $3 \mathrm{~min}$ in the convective cell-interiors and of 5-20 min in magnetic networks at the supergranular boundaries (Lites et al. 1993). In fact, signatures of upwardly propagating waves with their periods lying well within the range 3-20 min have recently been detected in solar coronal holes, even at a 
height of twice the solar radius above the solar limb (DeForest \& Gurman 1998; Ofman et al. 1999; Ofman et al. 2000a; Ofman et al. 2000b).

Given thus the expected periods of outwardlypropagating waves in solar wind flow-sheets, our aim here is to theoretically identify such waves and to calculate the approximate frequencies (which is also a measure of the wavelength in the $z$-direction; see for example, Kuijpers 1992; Cravens 1997) $f_{\mathrm{s}}=(2 \pi)^{-1}\left|k_{z}\right||655.0+c| \mathrm{Hz}$ of these waves, that may be measured in the in-situ observations by a spacecraft, or equivalently, by an observer fixed in the frame of the Sun. To perform such a task, it is required that we first examine the constraints that may be imposed on the possible magnitudes of such wavelengths from various physical and/or observational considerations.

Following a similar work by Hollweg (1982; see also discussions above) on surface waves in solar wind tangential discontinuities (TDs), we here assume that the waves are generated by motions in the $x-y$ plane in the photosphere/chromosphere of the Sun. In that case, the wavenumber $k_{y}$ can be regarded as determined by the spatial-scales of the solar granular/supergranular motions. Hollweg (1982) considered a transverse wavelength $\lambda_{y}=0.01 \mathrm{AU}$ that was obtained by extrapolating the mean distance between solar magnetic flux-tubes (Spruit $1981)$ out to $1 \mathrm{AU}$ under the assumption that the distance scales as $\left(B_{\mathrm{sun}} / B_{1 \mathrm{AU}}\right)^{1 / 2}$, with $B$ being the magnetic field-strength. We here consider this value $k_{y}=$ $2 \pi / \lambda_{y}=4.19 \times 10^{-6} \mathrm{~km}^{-1}$ for the wavenumber in the $y^{-}$ direction. For the maximum wavelength of magnetosonic waves in the $z$-direction, we again emperically consider a value $0.01 \mathrm{AU}$, as this is the approximate value for the observed correlation length of the fluctuating (or, wave) part of the magnetic field in solar wind (Barnes 1979; see also Cravens 1997). Although an explaination for the origin of such a correlation length cannot be given within the simple theoretical framework of the present paper, we still adapt this length as an upper limit for the magnitude of the longitudinal wavelength $\left|2 \pi / k_{z}\right|$ in our calculations without any further justification. With the observed average value for the half-width " $a$ " of the flow-sheets at $1 \mathrm{AU}$ being also approximately 0.01 AU (Thieme et al. 1990), we thus obtain (see Sect. 2)

$k_{y} a=2 \pi, k_{z} a \geq 2 \pi$, and $\theta \leq \pi / 4$,

with the periods of waves being in the range 3-20 min.

With the above bounds on the wave-parameters, we now turn to the numerical solutions of Eq. (7) to calculate the frequency bands (in the satellite frame) pertaining to various outwardly-propagating wavemodes that may present periodicities in the above range. Our numerical results suggest that the wavelengths (in the $z$-direction) of such waves are sufficiently small, so that, the angle of propagation for these waves are smaller than the critical angle $\theta_{\mathrm{c} 1}$ defined in Eq. (8) of Sect. 3. Waves that exist in this situation are (i) the forward-propagating magnetoacoustic slow surface waves (Fig. 1), (ii) the backward-propagating magnetoacoustic slow body waves
(Fig. 3) and (iii) the backward-propagating magnetoacoustic fast body and fast surface waves (Fig. 4). We here note that the forward-propagating slow surface waves and the backward-propagating fast surface waves were absent in the analysis of NRM96 as they confined themselves to the discussion of purely parallel propagation of waves in the flow-sheet.

In the satellite (or solar) frame and for periodicities in the range 3-20 $\mathrm{min}$, the forward-propagating magnetoacoustic slow surface waves present frequencies in a range $f_{\mathrm{s}}=6-40 \mathrm{mHz}$, while the backward-propagating magnetoacoustic (slow and fast) body and surface waves present frequencies in a range $20-100 \mathrm{mHz}$. The frequency band of $6-100 \mathrm{mHz}$ that we thus obtain are higher than the frequency band $\left(f_{\mathrm{s}}=10^{-5}-6.0 \times 10^{-3} \mathrm{~Hz}\right.$; see the review by $\mathrm{Tu} \&$ Marsch 1995) explored so far in search of magnetosonic waves in the high-speed solar wind streams. The results of such observations have often been negative, mostly showing the signatures of "PBS" $s$ in solar wind (e.g. Roberts 1990; Bavassano \& Bruno 1989, 1991; Tu \& Marsch 1991; Marsch \& Tu 1993). We however note, that Tu \& Marsch (1994) have recently attempted to explain some of the observed compressible fluctuations in $(2-5) \times 10^{-4} \mathrm{~Hz}$ frequency range in terms of a linear superposition of the "PBS"s with large-scale, transverse, fast magnetosonic waves in solar wind. In this paper, we consider a much simpler problem of the guided magnetosonic waves in an individual solar wind flow-sheet. The frequency range of $(4-5) \times 10^{-4} \mathrm{~Hz}$ may be presented in our calculations by the forward and backward-propagating surface and body waves with $k_{z} a=2 \pi$ and $\theta=\pi / 4$ that have periods in the range $3 \mathrm{hrs}$ to 3 days. Such long period waves may not be of solar origin, but may perhaps be generated in-situ by some istabilities, such as the parametric instabilities (e.g. Tu et al. 1989) or the instabilities pertaining to the backward-propagating, negative energy waves (see references in Sect. 1). We here note that obliquely propagating magnetosonic waves may also be generated non-linearly by Alfven wave phase mixing in an inhomogeneous plasma such as the solar wind flow-sheet with their rate of excitation being further modified in presence of steady shear flows; cf. Nakariakov et al. (1997, 1998).

In their calculations, NRM96 obtained standing magnetosonic body waves in the frame of the external flow with wavelengths that correspond to the critical wavenumbers $k_{\mathrm{z} 0}(\theta)$ (see Eq. (9), Sect. 3.1) at which the field-aligned phase-speed $c$ of the fundamental, backward-propagating, fast body kink and sausage waves drops to zero. In our numerical calculations, with $k_{y}=2 \pi / a$ for example, we obtain only one such stationary body mode that corresponds to the fundamental, backward-propagating, fast body sausage mode with a wavelength of about $9 \times 10^{5} \mathrm{~km}$ along the axis of the solar wind flow-sheet. We here note that, for $k_{y} \neq 0$, the numerical value of the critical wavelength pertaining to the fundamental, backwardpropagating, fast body kink mode is always longer than $0.01 \mathrm{AU}$, i.e. longer than the observed coherence length of 
magnetic fluctuations in solar wind flow (see discussions above). We therefore leave any further examination of such stationary fast body kink mode out of the present discussion. We however note, that in addition to the abovementioned stationary fast body sausage mode, two standing (kink and sausage) fast surface modes also appear in our calculations with $k_{y} a \geq 3.65 \pi$ that correspond to the backward-propagating, fast (kink and sausage) surface modes (see Fig. 4) with their critical wavenumbers $k_{\mathrm{z} 0}(\theta) a \geq 2 \pi$. Alongwith the standing fast body sausage wave, such stationary fast surface waves may possibly be detected in a frame co-moving with the ambient flow $\boldsymbol{U}_{\mathbf{e}}$ of the solar wind flow-sheet.

For extremely large longitudinal wavenumber $\left(k_{z} a>>\right.$ $2 \pi)$, but for a finite value of $k_{y} a(=2 \pi$, say) thus leading to $\theta<<\pi / 4$, we obtain a situation similar to the one discussed by NRM96, where the only surviving modes are the backward-propagating, magnetoacoustic slow and fast body (kink and sausage) modes (Figs. 3 and 4). Such backward body modes propagate in an outward direction with a common longitudinal phase-speed $c \approx 685 \mathrm{~km} \mathrm{~s}^{-1}$ in the frame of the Sun. All such backward body waves, however, propagate in a sunward (i.e., negative $z-$ ) direction with a common phase-speed of $v_{\mathrm{A} 0}$ as viewed in a frame comoving with the internal flow $\boldsymbol{U}_{\mathbf{0}}$ of the flow-sheet.

If, on the other hand, we consider an extremely large angle of propagation $\theta \rightarrow \pi / 2$ with respect to the flow vector $\boldsymbol{U}_{\mathbf{0}}$, while considering $k_{z} a(=2 \pi$, say $)$ to be finite thus implying $k_{y} a>>2 \pi$, the only outwardly-propagating wavemodes that we obtain are the forward-propagating (externally fast) magnetosonic slow and fast surface modes (Figs. 1 and 2). The phase-speed $c$ for both the kink and sausage slow surface modes is given by

$c=\left[\frac{\rho_{0}}{\rho_{0}+\rho_{\mathrm{e}}}\right] U_{0}+\left[\frac{\rho_{\mathrm{e}} v_{\mathrm{Ae}}^{2}+\rho_{0} v_{\mathrm{A} 0}^{2}}{\left(\rho_{\mathrm{e}}+\rho_{0}\right)}-\frac{\rho_{0} \rho_{\mathrm{e}}}{\left(\rho_{\mathrm{e}}+\rho_{0}\right)^{2}} U_{0}^{2}\right]^{1 / 2}$

in this limit of a very large angle of propagation $\theta$. We here note that, Eq. (14) coincides with the expression for the phase-speed of an incompressible magnetoacoustic surface wave propagating in the direction of the magnetic field in the presence of a parallel shear flow (Chandrasekhar 1961). In this limit of an angle of propagation $\theta \rightarrow \pi / 2$ and for a finite longitudinal wavevector $k_{z} a$, the field-aligned phase-speed $c$ for the outwardly-propagating fast surface modes approaches $\left(U_{0}+v_{\mathrm{c} 0}^{-}(\theta \rightarrow \pi / 2)\right) \approx\left(U_{0}+c_{\mathrm{T} 0}\right)$. For realistic numerical values of the physical parameters, Eq. (14) yields a phase-speed $c=780 \mathrm{~km} \mathrm{~s}^{-1}$ for the outwardly-propagating slow surface wave in the frame of the Sun. Similarly, the outwardly-propagating fast surface wave propagates with a longitudinal phase-speed $c=796 \mathrm{~km} \mathrm{~s}^{-1}$ with respect to the Sun in this limit of a very large angle of propagation $\theta \rightarrow \pi / 2$.

\subsection{Oppositely propagating waves}

As was pointed out in Sect. 3.2, earlier theoretical studies on waves in a solar wind fine structure did not obtain any magnetosonic waves propagating opposite to the solar wind flow even in a frame co-moving with the ambient flow external to the fine structure. Our numerical solution of the dispersion relations (7), that incorporates the effect of non-parallel propagation, however exhibits a number of such inwardly-propagating magnetoacoustic wavemodes in a fine solar wind flow-sheet. In the absence of any preferential length scales and time scales that may be intuitively associated with such waves, we may then arbitrarily consider the wavenumbers $k_{y} a=\left|k_{z} a\right|=2 \pi$ purely for the purpose of demonstration. In such a case, due to the existence of critical wavenumbers and the various upper and lower cut-offs (see Sect. 3.2), we obtain only one oppositely-propagating fundamental mode, namely, the (externally slow) magnetoacoustic fast body sausage mode (see Fig. 5) in the fine solar wind stream-structure with a period of approximately $15 \mathrm{hr}$ and a frequency of about $4.2 \times 10^{-4} \mathrm{~Hz}$ in the satellite (or solar) frame. This fast body sausage wave propagates with an inward phasespeed of $-27.6 \mathrm{~km} \mathrm{~s}^{-1}$ with respect to the external flow whereas, in a solar frame, it propagates with a phase velocity of $627.4 \mathrm{~km} \mathrm{~s}^{-1}$ in a direction away from the Sun.

In the case of an arbitrarily small angle of propagation $\theta<<\pi / 4$ with the transverse wavenumber $k_{y} a(=2 \pi$, say $)$ remaining finite, the existence of various cut-offs and critical wavenumbers ensure that no surface or fundamental body waves propagate in the flow-sheet in an inward direction in a frame co-moving with the external flow $\boldsymbol{U}_{\mathbf{e}}$. If, on the other hand, we consider a very large angle of propagation $\theta \rightarrow \pi / 2$, while keeping the magnitude for the longitudinal wavenumber $\left|k_{z} a\right|(=2 \pi$, say) to be finite thus leading to a very large transverse wavenumber $k_{y} a>>2 \pi$, we obtain three classes of oppositely-propagating fundamental wavemodes, namely, (i) the (externally slow) magnetoacoustic fast surface modes (Fig. 5), (ii) the (externally fast) magnetoacoustic fast surface modes (Fig. 7), and (iii) the (externally fast) magnetoacoustic fast body modes (Fig. 7).

Consider first the oppositely-propagating, (externally slow) fast surface modes. The longitudinal phase-speed $c$ for both these kink and sausage surface modes are given by

$c=\left[\frac{\rho_{0}}{\rho_{0}+\rho_{\mathrm{e}}}\right] U_{0}-\left[\frac{\rho_{\mathrm{e}} v_{\mathrm{Ae}}^{2}+\rho_{0} v_{\mathrm{A} 0}^{2}}{\left(\rho_{\mathrm{e}}+\rho_{0}\right)}-\frac{\rho_{0} \rho_{\mathrm{e}}}{\left(\rho_{\mathrm{e}}+\rho_{0}\right)^{2}} U_{0}^{2}\right]^{1 / 2}$

in this limit of an extremely large angle of propagation $\theta$ with respect to the vector $-\boldsymbol{U}_{\mathbf{0}}$. Equation (15) coincides with the expression for the phase-speed of an incompressible magnetohydrodynamic surface wave propagating in a direction opposite to the magnetic field in the presence of a parallel shear flow (Chandrasekhar 1961). Substitution of realistic numerical values for the physical parameters in a solar wind flow-sheet at $1 \mathrm{AU}$ yields $c \approx 650 \mathrm{~km} \mathrm{~s}^{-1}$ for the phase-speed of these oppositely-propagating (externally slow) magnetoacoustic fast surface waves in the frame of the Sun.

As $\theta \rightarrow \pi / 2$ with $\left|k_{z} a\right|=2 \pi$, the longitudinal phase speed $c$ for the oppositely-propagating (externally fast) 
fast surface modes approaches a limit $c=\left(U_{\mathrm{e}}-v_{\mathrm{ce}}^{-}(\theta)\right) \approx$ $\left(U_{\mathrm{e}}-c_{\mathrm{Te}}\right)$ that yields a numerical value of $c \approx 598 \mathrm{~km} \mathrm{~s}^{-1}$ in the frame of the Sun.

The oppositely-propagating, (externaly fast) magnetosonic, fast body kink and fast body sausage modes are of particular interest. For $\theta \rightarrow \pi / 2$ with $\left|k_{z} a\right|$ remaining finite, the longitudinal phase-speed $c$ of these inwardpropagating fast body modes can be given (in a frame co-moving with the external flow) by the following approximate formulae:

$\left|k_{z} a\right|=\frac{1}{N_{0}}\left\{\pi / 2+\tan ^{-1}\left[\left(\rho_{0} / \rho_{\mathrm{e}}\right) \frac{\Omega_{0}^{2} M_{\mathrm{e}}}{c^{2} N_{0}}\right]\right\}$

for the oppositely-propagating, fundamental, fast body kink mode; and

$\left|k_{z} a\right|=\frac{1}{N_{0}} \tan ^{-1}\left[\left(\rho_{0} / \rho_{\mathrm{e}}\right) \frac{\Omega_{0}^{2} M_{\mathrm{e}}}{c^{2} N_{0}}\right]$

for the oppositely-propagating, fundamental, fast body sausage mode. Here,

$N_{0}^{2} \approx\left(\Omega_{0}^{2}-2 v_{\mathrm{A} 0}^{2} \sec ^{2} \theta\right) / 2 v_{\mathrm{A} 0}^{2}$

and

$M_{\mathrm{e}}^{2} \approx\left(v_{\mathrm{Ae}}^{2} \sec ^{2} \theta-c^{2}\right) / v_{\mathrm{Ae}}^{2}$.

It can be shown, from Eqs. (16a)-(16d), that for a particular value of $\left|k_{z} a\right|$, the magnitude of the phase-speed $|c|$ exceeds the observed magnitude for the external flowspeed $U_{\mathrm{e}}$ as $\theta$ exceeds certain critical values, so that the fast body modes propagate towards the Sun even in a satellite (or solar) frame. The approximate value of such critical angles may be calculated from Eq. (16) after substituting $c=-U_{\mathrm{e}}=-655 \mathrm{~km} \mathrm{~s}^{-1}$. Our numerical solutions of the original dispersion relations (7) suggest that, for $\left|k_{z} a\right|=2 \pi$, such sunward-propagating magnetoacoustic, fast body kink and fast body sausage modes appear in a solar frame as the angle of propagation of the modes (with respect to the vector $-\boldsymbol{U}_{\mathbf{o}}$ ) approximately satisfies a relation $\theta \geq 82.97^{\circ}$. A possible mechanism for the in-situ excitation of such a sunward-propagating fast body wave in a solar wind flow-sheet may be the explosive instability in which the sunward-propagating wave interacts resonantly with a forward-propagating wave and a backwardpropagating wave so that the amplitude of all the three waves grow explosively in time; see Craik \& Adam (1979); Craik (1985); Nakariakov \& Oraevsky (1995); Nakariakov et al. (1996); Joarder et al. (1997b). A detailed examination of such a resonant triad of modes as well as the possibility of occurrence of the "explosive instability" in a solar wind fine structure is however complicated and is, therefore, beyond the scope of the present paper. We may also note that the sunward-propagating magnetosonic waves in solar wind, that are being discussed here, have not so far been detected in the inner-heliospheric observations by the space-satellites (cf. Roberts et al. 1987; see also the review by Kuijpers 1992). This is presumably because of the fact that the single-point observations of solar wind fluctuations, that have so far been carried out, were not adequate to detect the direction of phase propagation associated with wave motions in solar wind. It is to be hoped that the mutiple-point in-situ observations, such as the ones being undertaken by the recent Cluster-II mission (e.g. Escoubet et al. 2001), may shed some light on the direction of wave propagation in solar wind at a distance of 1 AU from the Sun.

Finally, it should be borne in mind that the linear magnetohydrodynamic wave theory, that we here apply to describe waves in a solar wind flow-structure, may not provide an accurate description for the solar wind plasma at a distance of $1 \mathrm{AU}$ from the Sun. A collisionless plasma theory employing Vlasov-Maxwell equations seems, on the other hand, to be more appropriate to describe fluctuations in plasma variables in solar wind flows (Barnes 1979). Such a kinetic description of solar wind plasma predicts strong "Landau damping" of certain wavemodes that is not captured in the MHD description of waves presented here. An appropriate description of the complex interaction of magnetosonic waves with the solar wind pressure balance structures, that are believed to give rise to the observed compressible fluctuations in solar wind (e.g. Tu \& Marsch 1994, 1995), is also complicated. Various specialised techniques, such as the "Pseudosound Theory" and the "NI-MHD Theory" (e.g. Montgomery et al. 1987) accompanied by computer simulations, are generally used to study the nature of such compressible fluctuations in solar wind.

In this paper, we, on the other hand, considered a simpler theoretical problem of propagation of linear magnetosonic waves in an isolated, model solar wind flow-sheet. The wavemodes that we find here must have their counterparts, perhaps in a much modified form, in more sophisticated theoretical treatments of the fluctuations of plasma variables in solar wind high-speed flows. Our analysis, therefore, may serve as a useful preliminary step towards the development of a more advanced theory to understand waves and turbulence in the inhomogeneous solar wind.

Acknowledgements. The author is indebted to Dr. V. M. Nakariakov for many helpful discussions and continuous encouragement. He also acknowledges useful comments of an anonymous referee on an earlier version of the paper that helped him in improving the content and presentation of the present version. He takes this opportunity to thank Dr. A. V. Thampan, Dr. Sonjoy Majumder and Mr. Baba Varghese for their help in the preparation of the manuscript. The earlier version of this paper was communicated by the author from the Indian Institute of Astrophysics, Bangalore, India-560034. Most of the revisions, leading to the present version, have been carried out during December, 2000 to July, 2001 when the author was a visiting scientist at the Inter-University Centre for Astronomy and Astrophysics (IUCAA), Pune, India -41100\%. The author thanks the Director, the scientists and the staff of IUCAA for providing much needed help and facilities. He is also thankful to the Director, VECC for offering him a fellowship for continuing research in solar magnetohydrodynamics. 


\section{References}

Andries, J., Tirry, W. J., \& Goossens, M. 2000, ApJ, 531, 561 Barnes, A. 1979, in Solar System Plasma Physics, 1, ed. C. F. Kennel, L. J. Lanzerotti, \& E. N. Parker (North-Holland, New York), 249

Bavassano, B. M., \& Bruno, R. 1989, J. Geophys. Res., 94, 1989

Bavassano, B. M., \& Bruno, R. 1991, J. Geophys. Res., 96, 1991

Burlaga, L. F. 1995, Interplanetary Magnetohydrodynamics (Oxford Univ. Press, New York), 8

Cairns, R. A. 1979, J. Fluid Mech., 92, 1

Chandrasekhar, S. 1961, Hydrodynamic and Hydromagnetic Stability (Oxford Clarendon Press, London)

Christensen-Dalsgaard, J. 1992, in The Sun: A Laboratory for Astrophysics, NATO ASI Ser. C: Mathematical and Physical Sciences, 373, ed. J. T. Schmelz, \& J. C. Brown (Kluwer, Dordrecht), 29

Craik, A. D. D. 1985, Wave Interaction and Fluid Flows (Cambridge Univ. Press, Cambridge)

Craik, A. D. D., \& Adam, J. A. 1979, J. Fluid Mech., 92, 15

Cravens, T. E. 1997, Physics of Solar System Plasmas (Cambridge Univ. Press, Cambridge)

DeForest, C. E., \& Gurman, J. B. 1998, ApJ, 501, L217

Del Zenna, L., \& Velli, M. 1994, in Solar Wind Nine, ed. H. R. Habbal, R. Esser, J. V. Hollweg, \& P. A. Isenberg, AIP Conf. Proc., No. 471

Edwin, P. M., \& Roberts, B. 1982, Sol. Phys., 76, 239

Edwin, P. M., \& Roberts, B. 1983, Sol. Phys., 88, 179

Escoubet, C. P., Fehringer, M., \& Bond, P. 2001, ESU Bull., 107, 43

Hollweg, J. V. 1982, J. Geophys. Res., 87, 8065

Hollweg, J. V., Yang, G., Cadez, V. M., \& Gakovic, B. 1990, ApJ, 349, 335

Jain, R., \& Roberts, B. 1991, Sol. Phys., 133, 263

Joarder, P. S., \& Roberts, B. 1992, A\&A, 256, 264

Joarder, P. S., \& Roberts, B. 1993, A\&A, 277, 225

Joarder, P. S., \& Satya Narayanan, A. 2000, A\&A, 359, 1211

Joarder, P. S., Nakariakov, V. M., \& Roberts, B. 1997a, Sol. Phys., 173, 81

Joarder, P. S., Nakariakov, V. M., \& Roberts, B. 1997b, Sol. Phys., 176, 285

Kuijpers, J. 1992, in ed. J. T. Schmelz, \& J. C. Brown, The Sun: A Laboratory for Astrophysics. NATO ASI Ser. C: Mathematical and Physical Sciences, 373 (Kluwer, Dordrecht), 535

Lites, B. W., Rutten, R. J., \& Kalkofen, W. 1993, ApJ, 414, 345

Mariani, F., Bavassano, B., Villante, U., \& Ness, N. F. 1973, J. Geophys. Res., 78, 8011

McCraken, K. G., \& Ness, N. F. 1966, J. Geophys. Res., 71, 3315

Mann, G., Marsch, E., \& Roberts, B. 1992, in Solar Wind Seven, COSPAR, ed. E. Marsch, \& R. Schwenn (Pergamon Press), 495

Marsch, E., \& Tu, C-Y. 1993, Ann. Geophys., 11, 659
Montgomery, D., Brown, M., \& Matthaeus, W. H. 1987, J. Geophys. Res., 92, 282

Nakariakov, V. M., \& Roberts, B. 1995, Sol. Phys., 159, 213

Nakariakov, V. M., \& Oraevsky, V. N. 1995, Sol. Phys., 160, 289

Nakariakov, V. M., Roberts, B., \& Mann, G. 1996, A\&A, 311, 311 (NRM96)

Nakariakov, V. M., Zugzhda, Y. D., \& Ulmschneider, P. 1996, A\&A, 312, 691

Nakariakov, V. M., Roberts, B., \& Murawski, K. 1997, Sol. Phys., 175, 93

Nakariakov, V. M., Roberts, B., \& Murawski, K. 1998, A\&A, 332,795

Nakariakov, V. M., Ofman, L., Deluca, E. E., Roberts, B., \& Davila, J. M., 1999, Science, 285, 862

Naugebaur, M. 1981, Fundam. Cosmic. Phys., 7, 131

Ofman, L., Nakariakov, V. M., \& DeForest, C. E. 1999, ApJ, 514,441

Ofman, L., Romoli, M., Poletto, G., Noci, G., \& Kohl, J. L. 2000a, ApJ, 529, 592

Ofman, L., Nakariakov, V. M., \& Sehgal, N. 2000b, ApJ, 533, 1071

Parker, E. N. 1963, Interplanetary Dynamical Processes (Interscience, New York)

Rae, I., \& Roberts, B. 1983, Sol. Phys., 84, 99

Roberts, B. 1981a, Sol. Phys., 69, 27

Roberts, B. 1981b, Sol. Phys., 69, 39

Roberts, B., \& Webb, A. R. 1979, Sol. Phys., 64, 77

Roberts, B., Edwin, P. M., \& Benz, A. O. 1984, ApJ, 279, 857

Roberts, D. A. 1990, J. Geophys. Res., 95, 1087

Roberts, D. A., Goldstein, M. L., Klein, L. W., \& Matthaeus, W. H. 1987, J. Geophys. Res., 92, 12023.

Ruderman, M. S., \& Goossens, M. 1995, J. Plasma Phys., 54, 149

Ruderman, M. S., \& Wright, A. N. 1998, J. Geophys. Res., 103(26), 573

Ruderman, M. S., Verwichte, E., Erdelyi, R., \& Goossens, M. 1996, J. Plasma Phys., 56, 285

Ryutova, M. P. 1988, Sov. Phys JETP, 67, 1594

Somasundaram, K., \& Uberoi, C. 1982, Sol. Phys., 81, 19

Spruit, H. C. 1981, in The Sun as a Star, CNRS/NASA Monograph Ser. on Nonthermal Phenomena in Stellar Atmospheres, ed. S. Jordan, NASA SP-450, 385

Spruit, H. C., \& Roberts, B. 1983, Nature, 304, 401

Thieme, K. M., Marsch, E., \& Schwenn, R. 1990, Ann. Geophys., 8, 713

Tirry, W., Cadez, V., Erdeyli, R., \& Goossens, M. 1998, A\&A, 332,786

Tu, C-Y., \& Marsch, E. 1991, Ann. Geophys., 9, 319

Tu, C-Y., \& Marsch, E. 1994, J. Geophys. Res., 99(21), 481

Tu, C-Y., \& Marsch, E. 1995, Space Sc. Rev., 73, 1

Tu, C-Y., Marsch, E., \& Thieme, K. M. 1989, J. Geophys. Res., 94, 11739

Uberoi, C. 1982, Sol. Phys., 78, 351

Velli, M., Habbal, S. R., \& Esser, R. 1994, Space Sc. Rev., 70, 391 